\title{
Enhanced anticancer properties of lomustine in conjunction with docosahexaenoic acid in glioblastoma cell lines
}

\author{
Kevin A. Harvey, BS, ${ }^{1}$ Zhidong Xu, PhD, ${ }^{1}$ M. Reza Saaddatzadeh, PhD, ${ }^{2,4}$ Haiyan Wang, MD, ${ }^{2}$ \\ Karen Pollok, PhD, ,3 Aaron A. Cohen-Gadol, MD, MSc, ${ }^{4}$ and Rafat A. Siddiqui, PhD ${ }^{1,5}$ \\ ${ }^{1}$ Cellular Biochemistry Laboratory, Indiana University Health Methodist Research Institute; ${ }^{2 H e r m a n ~ B . ~ W e l l s ~ C e n t e r ~ f o r ~ P e d i a t r i c ~}$ \\ Research, Department of Pediatrics, Section of Pediatric Hematology/Oncology, Riley Hospital for Children at Indiana University \\ Health; ${ }^{3}$ Department of Pharmacology and Toxicology and ${ }^{4}$ Goodman Campbell Brain and Spine, Department of Neurological \\ Surgery, Indiana University School of Medicine; and ${ }^{5}$ Department of Medicine, Indiana University School of Medicine, \\ Indianapolis, Indiana
}

OBJECT Glioblastoma is a rapidly infiltrating tumor that consistently rematerializes despite various forms of aggressive treatment. Brain tumors are commonly treated with alkylating drugs, such as lomustine, which are chemotherapeutic agents. Use of these drugs, however, is associated with serious side effects. To reduce the side effects, one approach is to combine lower doses of chemotherapeutic drugs with other nontoxic anticancer agents. In this study, using glioblastoma cell lines, the authors investigated the anticancer effects of lomustine, alone and in combination with docosahexaenoic acid (DHA), an omega-3 polyunsaturated fatty acid normally abundant in the brain and known for its anticancer potential.

METHODS Cells were cultured from 3 human-derived tumor cell lines (U87-MG, DB029, and MHBT161) and supplemented with either DHA or lomustine to determine the growth inhibitory potential using WST-1, a mitochondrial functional indicator. Human-derived cerebral cortex microvascular endothelial cells served as a normal phenotypic control. Cellular incorporation of DHA was analyzed by gas chromatography. Using flow cytometric analysis, the DHA and/or lomustine effect on induction of apoptosis and/or necrosis was quantified; subsequently, the DHA and lomustine effect on cell cycle progression was also assessed. Western blot analysis confirmed the role of downstream cellular targets.

RESULTS U87-MG growth was inhibited with the supplementation of either $\mathrm{DHA}\left(\mathrm{ED}_{50} 68.3 \mu \mathrm{M}\right)$ or lomustine $\left(\mathrm{ED}_{50}\right.$ $68.1 \mu \mathrm{M})$; however, growth inhibition was enhanced when U87-MG cells were administered equimolar doses of each compound, resulting in nearly total growth inhibition at $50 \mu \mathrm{M}$. Gas chromatography analysis of the fatty acid profile in DHA-supplemented U87-MG cells resulted in a linear dose-dependent increase in DHA incorporation $(<60 \mu \mathrm{M})$. The combination of DHA and lomustine potently induced U87-MG apoptosis and necrosis as indicated by flow cytometric analysis. Activation of caspase-3 and poly (ADP-ribose) polymerase (PARP) was evident in lomustine-treated U87-MG cells, although this activation did not appear to be dependent on DHA supplementation. Additionally, lomustine-treated cells' growth arrested in the $\mathrm{G}_{2} / \mathrm{M}$ cell cycle stage, regardless of the presence of DHA. Similar to the U87-MG observations, the combination of DHA and lomustine resulted in growth inhibition of 2 additional human-derived glioblastoma cell lines, DB029 and MHBT161. Importantly, in primary human-derived cerebral cortex endothelial cells, this combination was only growth inhibitory $(40.8 \%)$ at the highest dose screened $(100 \mu \mathrm{M})$, which indicates a certain degree of selectivity toward glioblastoma.

CONCLUSIONS Taken together, these data suggest a potential role for a combination therapy of lomustine and DHA for the treatment of glioblastomas.

http://thejns.org/doi/abs/10.3171/2014.10.JNS14759

KEY WORDS docosahexaenoic acid; lomustine; glioblastoma; brain cancer; oncology

\footnotetext{
ABBREVIATIONS BCA = bicinchoninic acid; CSC = Cell Systems Corporation; DHA = docosahexaenoic acid; DMEM = Dulbecco's modified essential medium; DPA = docosapentaenoic acid; $\mathrm{ED}_{50}=$ median effective dose; $\mathrm{EMEM}$ = Eagle's minimum essential medium; $\mathrm{EPA}=$ eicosapentaenoic acid; $\mathrm{FBS}=$ fetal bovine serum; $\mathrm{HBMEC}=$ human brain microvascular endothelial cell; HCCMEC = human cerebral cortex microvascular endothelial cell; OD = optical density; PARP = poly (ADP-ribose) polymerase; PBS = phosphate-buffered saline; PUFA = polyunsaturated fatty acid.

SUBMITTED April 3, 2014. ACCEPTED October 22, 2014.

INCLUDE WHEN CITING Published online December 19, 2014; DOI: 10.3171/2014.10.JNS14759.

DISCLOSURE The authors report no conflicts of interest concerning the materials or methods used in this study and the findings specified in this paper.
} 
$\mathrm{G}$ LIOBLASTOMA, the most common and malignant form of cancer in the central nervous system, is characterized either as primary, which is a rapidly progressing tumor that typically translates into an unfavorable clinical prognosis, or secondary, which presumably develops slowly from a low-grade diffuse astrocytoma. ${ }^{27}$ Since aggressive primary tumors are highly infiltrative, tumor recurrence is frequent despite resection and aggressive treatment. Patients diagnosed with glioblastoma have a median survival of approximately 15 months; 2 years after diagnosis, the survival rate is $27 \% .{ }^{17,41}$ Long-term survival rates of patients with glioblastoma have been extended with a combination treatment of radiotherapy, lomustine, and temozolomide. ${ }^{11}$ Both progression-free and overall survival rates increased, but acute toxicity remained a significant factor. Lomustine is a lipid-soluble alkylating agent that is capable of crossing through the blood-brain barrier. It acts nonspecifically and can dramatically affect rapidly dividing normal cells in addition to the targeted tumor cells. To reduce the toxicity associated with high doses of lomustine, investigators have used nanoparticle technology to more efficiently deliver lower effective doses to the cellular target. ${ }^{23,24}$ Numerous clinical trials have revolved around the use of lomustine or temozolomide in conjunction with additional therapeutic reagents, including, but not limited to, antibodies and pharmacological inhibitors..$^{2,43,45}$ Despite these attempts to markedly improve clinical outcomes, relatively few gains have been made in extending survival curves or drastically reducing the cytotoxicity associated with the use of these chemotherapeutic reagents.

Docosahexaenoic acid (DHA), an omega-3 polyunsaturated fatty acid (PUFA), has widely accepted health benefits, ${ }^{38}$ furthermore, previous investigations performed in our laboratory have enabled us to extensively describe the role of DHA in preventing and treating cancers in vitro and in vivo. ${ }^{34,36,37}$ DHA and arachidonic acid, an omega-6 fatty acid, are the most abundant PUFAs in the brain. Interestingly, these fatty acids have been shown to have opposing effects on tumor growth and cell migration. ${ }^{19,26}$ When the balance of fatty acid content favors arachidonic acid, a promigratory tumor cell phenotype emerges; however, if these tumor cells are rich in DHA, their migratory potential will be reduced, suggesting that omega-3 fatty acid may inhibit the infiltrative potential of aggressive glioblastomas. ${ }^{7,26}$ This migratory balance is mediated by brain fatty acid-binding protein (FABP) expression. Faragó et al. demonstrated the effect of PUFAs, including DHA, on the involvement of the microRNA profile in apoptosis-specific changes in treated glioblastoma cell lines. ${ }^{8}$ In their study, these investigators observed similar findings with the administration of temozolomide alone.

Conjugation of molecules directly to DHA or use of a secondary compound in conjunction with DHA as an anticancer approach has been previously explored. ${ }^{12,34,39}$ These approaches were designed either to increase efficacy or enhance the specificity of targeted cells. Wang et al. combined the use of DHA with the anticancer drug etoposide (VP16) to induce apoptosis in medulloblastoma cell lines, Daoy and D283, and glioblastoma cell lines, U87 and $\mathrm{U} 138 .{ }^{44} \mathrm{U} 87$ and $\mathrm{U} 138$ cell lines were insensitive to pretreatment with DHA followed by VP16 administration, whereas Daoy and D283 cells showed little responsiveness to either treatment alone, but a significant induction of apoptosis was observed with the combination. Wang's evidence suggests that the apoptosis mechanism involves the downregulation of survival pathways associated with DNA repair and PI3K/MAPK. In this study, we investigated the anticancer effects of lomustine, alone or in combination with DHA, on glioblastoma cell lines. To our knowledge, this is the first demonstration of the combined use of lomustine and DHA as an anticancer strategy.

\section{Methods \\ Materials}

U87-MG, a human-derived glioblastoma-astrocytoma cell line, and Eagle's minimum essential medium (EMEM) were purchased from ATCC. Human brain microvascular endothelial cells, Cell Systems Corporation (CSC) complete medium, and CultureBoost-R recombinant growth factor supplements were acquired from Cell Systems Corporation. Cells from the DB029 cell line (a human-derived Grade 3 glioblastoma cell line) were a gift from Dr. John Bright (Indiana University Health Methodist Research Institute). The MHBT161 glioblastoma cell line was developed from tumor resected from a patient by Dr. Aaron Cohen-Gadol (using Indiana University IRBapproved protocols) and processed by Drs. Karen Pollok and M. Reza Saadatzadeh. The Indiana University Institutional Review Board approved the study, and informed consent was obtained from the patient. Fetal bovine serum (FBS), Dulbecco's modified essential medium (DMEM), and all electrophoresis products were purchased from Life Technologies. Lomustine, temozolomide, and additional chemicals were acquired from Sigma Chemical Company. Consumable tissue culture products were obtained from Fisher Scientific. The Annexin V FLUOS staining kits were purchased from Roche Applied Science. Gas chromatography standards were acquired from Restek Corporation. Fatty acids were obtained from Nu-Chek Prep. Western blot antibodies were purchased from Cell Signaling Technology, unless otherwise noted.

\section{Cell Culture}

U87-MG cells were maintained in EMEM supplemented with $10 \%$ FBS and $1 \%$ antibiotic-antimycotic solution. DB029 and MHBT161 cell lines were cultured in DMEM also supplemented with $10 \%$ FBS and $1 \%$ antibiotic-antimycotic solution. Human brain microvascular endothelial cells (HBMECs), a primary cell line, were maintained in CSC complete medium supplemented with $10 \%$ FBS and CultureBoost-R recombinant growth factors as described by the manufacturer. All cell lines were maintained at $37^{\circ} \mathrm{C}$ in $5 \% \mathrm{CO}_{2}$ in a humidified atmosphere.

\section{Cell Proliferation Assay}

Initially, cells (5000 cells/well) were cultured in 96well flat-bottom plates overnight in complete medium to establish a linear growth rate. Spent medium was replaced with new medium supplemented with $2 \%$ FBS and varying treatments (100 $\mu$ l total volume/well). Ethanol-supplemented cells $(<0.5 \%)$ served as the vehicle control. Cells were maintained at $37^{\circ} \mathrm{C}$ in $5 \% \mathrm{CO}_{2}$ in a humidified at- 
mosphere for 24 hours prior to assessment of cell growth with the WST-1 assay reagent (Roche Applied Science) as described by the manufacturer. Medium alone combined with the WST-1 assay reagent established nonspecific values that were subtracted from the experimental optical density (OD) readings (OD at $450 \mathrm{~nm}$ ). Vehicle control OD readings served as standard proliferative potential normalized to $100 \%$. The proliferation index was calculated by dividing the average OD treatment reading by the average OD vehicle reading and multiplying by 100 .

\section{Analysis of Fatty Acid Incorporation by Gas Chromatography}

Subconfluent cells were cultured in complete medium in the absence or presence of varying doses of DHA for 24 hours under standard tissue culture conditions. Following the incubation, cells were trypsinized and washed twice in calcium- and magnesium-free phosphate-buffered saline (PBS) supplemented with $1 \%$ fatty acid-free bovine serum albumin. Cell pellets were resuspended in PBS and sonicated to lyse the cells, and an internal standard (C23:0) was added to a portion of the cell lysates. Using each of the remaining lysates, the protein concentration was quantified using a bicinchoninic acid (BCA) protein assay kit (Pierce) to normalize the assessed amount of fatty acids to protein content.

Using the Folch method, ${ }^{9}$ lipids were extracted from the cell lysates with chloroform:methanol $(2: 1, \mathrm{v} / \mathrm{v})$. The fatty acids were transesterified using a modified procedure from Lepage and Roy. ${ }^{20}$ Briefly, $2 \mathrm{ml}$ of methanol:benzene (4:1, $\mathrm{v} / \mathrm{v}$ ) was added to each nitrogen-dried sample and cooled in a dry ice bath for 10 minutes followed by the addition of $200 \mu \mathrm{l}$ of acetyl chloride. Transesterification occurred at room temperature over a 24-hour incubation. The addition of $6 \% \mathrm{~K}_{2} \mathrm{CO}_{3}$ neutralized the reaction. Following centrifugation, the benzene layer was recovered and subjected to separation on a gas chromatography system (Shimadzu GC2010) equipped with a Zebron ZB-WAXplus column (100 m, $0.25 \mathrm{~mm}$ ID, $0.25 \mu \mathrm{m}$ thickness). The column temperature program was as follows: temperature was held at $30^{\circ} \mathrm{C}$ for 2 minutes, increased to $180^{\circ} \mathrm{C}$ at $20^{\circ} \mathrm{C}$ per minute, held at $180^{\circ} \mathrm{C}$ for 2 minutes, increased to $207^{\circ} \mathrm{C}$ at $4^{\circ} \mathrm{C}$ per minute, held at $207^{\circ} \mathrm{C}$ for 3 minutes, increased to $220^{\circ} \mathrm{C}$ at $2^{\circ} \mathrm{C}$ per minute, held at $220^{\circ} \mathrm{C}$ for 2 minutes, and then increased to $240^{\circ} \mathrm{C}$ at $2{ }^{\circ} \mathrm{C}$ per minute before finally being held at $240^{\circ} \mathrm{C}$ for 2 minutes. Fatty acid peaks were detected using a flame ionization detector at $250^{\circ} \mathrm{C}$, and peak identification was achieved by comparing the results to the retention times of authentic fatty acid standards. Data were analyzed with Shimadzu's GC Solutions software. Fatty acid quantities were normalized based on the internal standard (C23:0) and calculated based on an external standard curve in conjunction with protein concentrations.

\section{Apoptosis Detection}

Cells $\left(2.5 \times 10^{5}\right)$ were plated in complete medium overnight in 6-well tissue culture treated plates. Spent medium was replaced with $3 \mathrm{ml}$ of EMEM supplemented with $2 \%$ FBS and varying treatment conditions. Cells were maintained in standard tissue culture conditions for 24 hours. All spent medium, PBS washes, and trypsinized cells were combined to ensure that no cell loss occurred during har- vesting. Cell pellets were resuspended in an Annexin V FLUOS/propidium iodide labeling solution as described by the manufacturer. Labeling occurred in the dark at room temperature for 20 minutes. Analysis was performed on a FACSCalibur flow cytometer (Becton Dickinson) equipped with a $15-\mathrm{mW}$ air-cooled argon-ion laser emitting at a 488$\mathrm{nm}$ wavelength. Annexin V FLUOS, which binds phosphatidylserine on the plasma membrane, was detected through a 530-nm band pass filter. Propidium iodide, which binds DNA by passing through the compromised membranes, was detected through a 650-nm long pass filter. Apoptotic cells bind Annexin V FLUOS on their outer plasma membrane, whereas necrotic cells are distinguished by the additional propidium iodide incorporation. Viable cells do not display any fluorescent indicators. Data were quantified as a percentage of total cells, excluding cellular debris, using CellQuest software (Becton Dickinson).

\section{Western Blot Analysis}

Subconfluent U87-MG cells were grown in 6-well tissue culture treated plates and treated with DHA, lomustine, or a combination of the two in EMEM complete medium, containing $2 \%$ FBS for 24 hours under standard tissue culture conditions. Treated cells were rinsed in cold PBS and lysed on ice for 15 minutes in a radioimmunoprecipitation assay (RIPA) lysis buffer (Millipore) containing $100 \mathrm{mM}$ $\mathrm{NaF}, 2 \mathrm{mM} \mathrm{Na} \mathrm{VO}_{4}, 2.5 \mathrm{mM}$ diisopropyl fluorophosphate, and complete mini protease cocktail inhibitor tablets (Roche Applied Science). Following sample centrifugation to remove the insoluble matter from the detergent solubilized extracts, the protein content was quantified using a BCA protein assay kit. Proteins were linearized by heating at $100^{\circ} \mathrm{C}$ for 10 minutes; subsequently, proteins were electrophoretically separated in $4 \%-12 \%$ polyacrylamide gradient gels and transferred onto nitrocellulose membranes. Membranes were blocked for 30 minutes at room temperature in Tris-buffered saline supplemented with $0.1 \%$ Triton X-100 (TBST) and 10\% Roche Western blocking reagent. Blots were probed with primary antibodies in accordance with the manufacturer's recommendations. Secondary antibodies were peroxidase-conjugated for protein detection using an enhanced chemiluminescence (ECL) system (Amersham Pharmacia Biotechnology). Nitrocellulose membranes were stripped in Restore Western blot stripping buffer (Thermo Scientific) for 15 minutes at room temperature. Stripped blots were washed 6 times in TBST, blocked, and reprobed with an alternative antibody.

\section{Cell Cycle Analysis}

U87-MG cells $\left(3.5 \times 10^{5}\right)$ were plated and maintained in EMEM complete medium overnight in 6-well tissue culture treated plates. To synchronize the cell cycle status of the U87-MG cells, spent medium was replaced with EMEM supplemented with $0.2 \%$ FBS for 24 hours under standard tissue culture conditions. Cells were treated for 48 hours in EMEM containing 2\% FBS. Following treatment, the cells were trypsinized and washed in PBS; the resulting cell pellets were resuspended in $0.5 \mathrm{ml}$ of PBS. Ice-cold $70 \%$ ethanol solution $(4.5 \mathrm{ml})$ was added to fix the cell suspensions, which were stored at $-20^{\circ} \mathrm{C}$ until cells were labeled and analyzed. The cells were washed in PBS 
prior to being resuspended in labeling solution, which consisted of PBS supplemented with $0.1 \%$ Triton X-100, 0.2 $\mu \mathrm{g}$ of DNase-free RNase A, and $20 \mu \mathrm{g} / \mathrm{ml}$ of propidium iodide. Labeling occurred for 30 minutes at room temperature in the dark. Analysis was performed on a FACSCalibur flow cytometer (Becton Dickinson) equipped with a $15-\mathrm{mW}$ air-cooled argon-ion laser emitting at a 488-nm wavelength. Propidium iodide, which binds DNA by passing through the compromised membranes, was detected through a $650-\mathrm{nm}$ long pass filter. A gate was established surrounding targeted events to ensure doublet and nonviable cell discrimination. Determination of cell cycle status was assessed using ModFit LT Software (Becton Dickinson). Results are expressed as the mean \pm SD of 3 determinants.

\section{Statistical Analysis}

Data are reported as mean $\pm \mathrm{SD}$. For apoptosis/necrosis and proliferation measures, comparisons among different treatments and comparison with control were performed using the Student t-test. To adjust for multiple comparisons, the Bonferroni correction was used. For example, if 3 separate treatment doses were compared with control in a single experiment, only $\mathrm{p}$ values $\leq 0.017$ (i.e., $0.05 / 3$ ) were considered significant.

\section{Results}

\section{Effect of Fatty Acid Supplementation and Chemotherapeutic Reagents on Growth Inhibition}

Figure 1A depicts the relative proliferative potential of long-chain omega-3 fatty acid-supplemented cells following a 24-hour treatment. All three of the omega-3 fatty acids used in this study effectively demonstrated a significant impact on U87-MG cell growth: docosahexaenoic acid (DHA; median effective dose $\left[\mathrm{ED}_{50}\right] 68.3 \mu \mathrm{M}$ ), eicosapentaenoic acid (EPA; $\left.\mathrm{ED}_{50} 63.5 \mu \mathrm{M}\right)$, and docosapentaenoic acid (DPA; $\left.\mathrm{ED}_{50} 51.4 \mu \mathrm{M}\right)$. Since the most prominent omega-3 fatty acid in neurological tissue is DHA, additional experiments focused solely on this fatty acid. Data presented in Fig. 1B portray the effect of individual fatty acids representing other fatty acid classes, including the omega- 6 fatty acids arachidonic acid and linoleic acid; an omega-9 fatty acid, oleic acid; and saturated palmitic acid. While linoleic and palmitic acids did produce growth inhibition at concentrations greater than $70 \mu \mathrm{M}$, none of these fatty acids inhibited proliferation by more than $40 \%$ at concentrations up to $100 \mu \mathrm{M}$. The effect of the chemotherapeutic reagents, temozolomide and lomustine, on U87-MG cell proliferation is shown in Fig. 1C. Temozolomide negatively affected proliferation at doses greater than $50 \mu \mathrm{M}$; however, the impact was relatively inconsequential. Lomustine demonstrated a dose-dependent decrease in U87-MG cell proliferation with $80 \%$ growth inhibition observed at $100 \mu \mathrm{M}\left(\mathrm{ED}_{50} 68.1 \mu \mathrm{M}\right)$.

\section{U87-MG Cellular Incorporation of DHA}

DHA was incorporated in a dose-dependent manner at doses up to $60 \mu \mathrm{M}$, which was the maximum dose assessed due to significant cell loss at higher concentrations (Table 1). Corresponding with the increasing doses of DHA, relative decreases were also observed in levels of
A

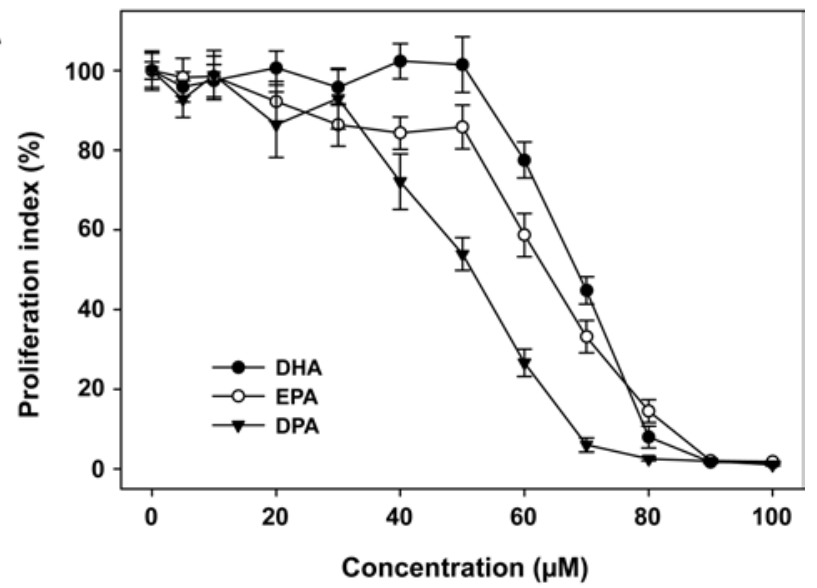

B
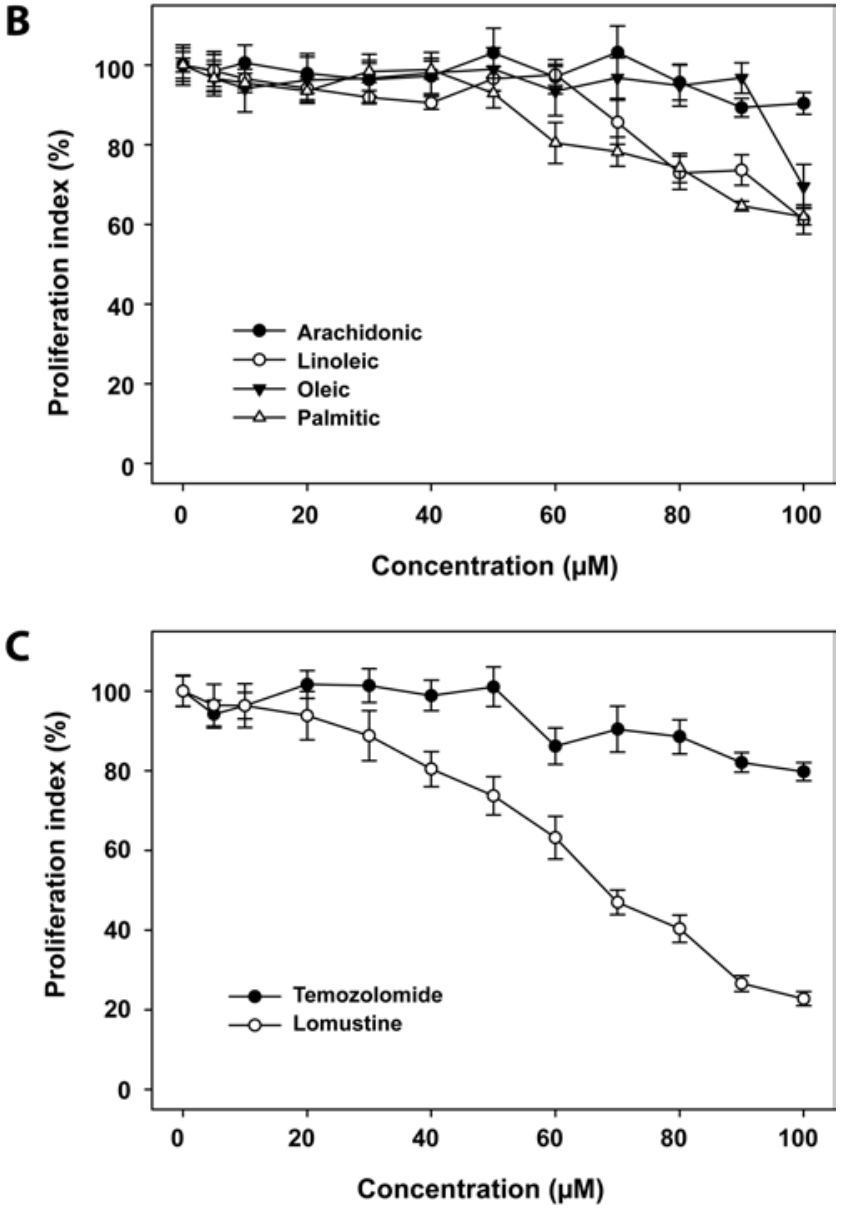

FIG. 1. Effect of fatty acid supplementation on U87-MG growth inhibition. Cells were supplemented with varying concentrations of individual fatty acids and cultured for 24 hours as described in Methods. WST-1 was administered to assess cell growth. Vehicle controls were used to calculate the growth rate of untreated cells $(100 \%)$ while media-only wells permitted the subtraction adjustment for background readings (0\%). A: Data represent the three omega-3 fatty acids tested. B: Representatives from omega-6 PUFAs (arachidonic and linoleic acids), an omega-9 monounsaturated fatty acid (oleic acid), and a saturated fatty acid (palmitic acid) were assessed for their growth inhibitory potential. C: Effect of the chemotherapeutic reagents temozolomide and lomustine on U87-MG growth inhibition. Results are expressed as the mean \pm SD of 4 determinants. 
nearly every identified fatty acid; however, these data can be misleading when only the percentages of fatty acids are reported. As shown in Table 2, when the fatty acid distribution is reported as absolute quantities, the results take on a much different interpretation. For example, the actual amount of stearic and oleic acids present in the cells remained relatively unchanged in the presence of varying doses of DHA, while the incorporation of palmitic acid significantly increased. The relative percentage of stearic and oleic acids appears to indicate a loss in these fatty acids via utilization and/or conversion, but analysis of the actual quantities of these fatty acids that were present in the cells clearly shows that no loss occurred.

\section{Growth Inhibitory Effect of DHA and Lomustine Combination}

As shown in Fig. 2, treatment with the combination of DHA and lomustine dramatically inhibited the proliferative potential of U87-MG cells. The combination effect was dose dependent; furthermore, growth inhibition was observed at a concentration as low as $10 \mu \mathrm{M}$ within the 24-hour treatment. Visual inspection of these cell cultures indicated that fewer cells were present, which was consistent with cell loss in addition to the growth inhibition (data not shown).

\section{Effect of DHA and Lomustine on U87-MG Cell Apoptosis/ Necrosis}

Since significant growth inhibition was observed, particularly in the presence of the combination of DHA and lomustine, the effect on U87-MG cell viability was investigated. Data shown in Table 3 summarize these findings. Vehicle control cells consisted of $90.2 \% \pm 1.5 \%$ viable, $7.0 \% \pm 1.1 \%$ apoptotic, and $2.8 \% \pm 0.5 \%$ necrotic cell populations, which was a predictable diversity based on limited cell loss during routine tissue culture maintenance. Cells supplemented with DHA $(30$ and $40 \mu \mathrm{M})$ consistently mirrored the profile of control cells, with only slight increases in apoptosis and necrosis. Although a statistically significant shift in U87-MG cell viability was apparent, most of the cells treated with lomustine $(30$ and $40 \mu \mathrm{M})$ were viable. Cell viability significantly diminished when the U87-MG cells were supplemented with both DHA and lomustine, resulting in only $23.1 \%$ viability at the $40 \mu \mathrm{M}$ combined dose. The effects of lomustine in the absence or presence of DHA were further analyzed for caspase-3 activation. As shown in Fig. 3, decreases in caspase-3 and poly (ADP-ribose) polymerase (PARP) protein expression levels were diminished in cells supplemented with lomustine alone or in combination with DHA. Furthermore, cleavedPARP detection corresponded to samples that had diminished full-length PARP expression. This evidence supports the observations from the flow cytometric data analysis on lomustine/DHA-enhanced cellular apoptosis.

\section{Effect of DHA and Lomustine on U87-MG Cell Cycle Status}

Table 4 depicts the percentage of viable cells within each cell cycle stage. The distribution of DHA-supplemented cells closely resembled the population distribution in the vehicle control. Both lomustine and the combination

TABLE 1. Relative percentage of targeted fatty acids in DHA-supplemented U87-MG cells*

\begin{tabular}{|c|c|c|c|c|c|c|c|}
\hline $\mathrm{FA}$ & Vehicle & $10 \mathrm{mM}$ & $20 \mathrm{mM}$ & $30 \mathrm{mM}$ & $40 \mathrm{mM}$ & $50 \mathrm{mM}$ & $60 \mathrm{mM}$ \\
\hline $12: 0$ & $0.11 \pm 0.00$ & $0.09 \pm 0.01$ & $0.06 \pm 0.00$ & $0.26 \pm 0.01$ & $0.05 \pm 0.00$ & $0.04 \pm 0.00$ & $0.04 \pm 0.00$ \\
\hline $14: 0$ & $1.60 \pm 0.17$ & $1.57 \pm 0.03$ & $1.56 \pm 0.09$ & $1.38 \pm 0.07$ & $1.37 \pm 0.03$ & $1.30 \pm 0.06$ & $1.17 \pm 0.02$ \\
\hline 16:0 (palmitic acid) & $25.23 \pm 3.30$ & $24.29 \pm 0.67$ & $21.55 \pm 1.05$ & $19.53 \pm 0.33$ & $21.40 \pm 1.05$ & $20.42 \pm 2.13$ & $19.14 \pm 0.26$ \\
\hline $16: 1 n-7$ & $2.57 \pm 0.30$ & $1.66 \pm 0.02$ & $1.44 \pm 0.09$ & $1.23 \pm 0.07$ & $1.14 \pm 0.01$ & $1.09 \pm 0.03$ & $0.81 \pm 0.14$ \\
\hline 18:0 (stearic acid) & $17.99 \pm 1.53$ & $17.46 \pm 0.69$ & $16.23 \pm 0.86$ & $13.52 \pm 0.80$ & $12.11 \pm 0.11$ & $11.46 \pm 0.35$ & $9.80 \pm 0.24$ \\
\hline 18:1n-9 (oleic acid) & $25.65 \pm 2.18$ & $20.52 \pm 0.74$ & $17.55 \pm 0.96$ & $14.51 \pm 0.85$ & $13.25 \pm 0.10$ & $12.46 \pm 0.37$ & $10.89 \pm 0.29$ \\
\hline 18:2n-6 (linoleic acid) & $4.59 \pm 0.38$ & $4.10 \pm 0.12$ & $3.57 \pm 0.21$ & $3.00 \pm 0.17$ & $2.79 \pm 0.02$ & $2.59 \pm 0.07$ & $2.32 \pm 0.07$ \\
\hline $18: 3 n-6$ & $0.09 \pm 0.00$ & $0.09 \pm 0.00$ & $0.08 \pm 0.01$ & $0.06 \pm 0.00$ & $0.06 \pm 0.00$ & $0.05 \pm 0.00$ & $0.05 \pm 0.00$ \\
\hline $18: 3 n-3$ & $0.09 \pm 0.01$ & $0.06 \pm 0.01$ & $0.10 \pm 0.01$ & $0.09 \pm 0.00$ & $0.11 \pm 0.02$ & $0.10 \pm 0.02$ & $0.08 \pm 0.00$ \\
\hline $18: 4 n-3$ & $0.64 \pm 0.07$ & $0.41 \pm 0.01$ & $0.35 \pm 0.03$ & $0.33 \pm 0.02$ & $0.29 \pm 0.01$ & $0.30 \pm 0.00$ & $0.25 \pm 0.02$ \\
\hline $20: 0$ & $0.34 \pm 0.03$ & $0.27 \pm 0.02$ & $0.25 \pm 0.01$ & $0.22 \pm 0.01$ & $0.22 \pm 0.01$ & $0.20 \pm 0.02$ & $0.19 \pm 0.02$ \\
\hline $20: 4 n-6(A A)$ & $9.21 \pm 0.69$ & $7.98 \pm 0.35$ & $7.26 \pm 0.32$ & $6.02 \pm 0.36$ & $5.25 \pm 0.04$ & $5.07 \pm 0.16$ & $4.31 \pm 0.11$ \\
\hline $20: 5 n-3$ (EPA) & $0.63 \pm 0.01$ & $0.98 \pm 0.05$ & $1.57 \pm 0.10$ & $1.52 \pm 0.07$ & $1.46 \pm 0.01$ & $1.42 \pm 0.03$ & $1.26 \pm 0.03$ \\
\hline $22: 0$ & $0.37 \pm 0.02$ & $0.28 \pm 0.02$ & $0.24 \pm 0.01$ & $0.21 \pm 0.01$ & $0.18 \pm 0.00$ & $0.17 \pm 0.01$ & $0.14 \pm 0.01$ \\
\hline $22: 4 n-6$ & $3.02 \pm 0.15$ & $2.71 \pm 0.13$ & $2.50 \pm 0.11$ & $2.30 \pm 0.13$ & $1.87 \pm 0.02$ & $1.86 \pm 0.03$ & $1.68 \pm 0.03$ \\
\hline $22: 5 n-6$ & $0.34 \pm 0.01$ & $0.30 \pm 0.02$ & $0.48 \pm 0.11$ & $0.64 \pm 0.17$ & $0.63 \pm 0.21$ & $0.59 \pm 0.18$ & $0.42 \pm 0.12$ \\
\hline $22: 5 n-3$ (DPA) & $2.25 \pm 0.11$ & $2.15 \pm 0.09$ & $2.23 \pm 0.09$ & $2.02 \pm 0.11$ & $1.75 \pm 0.02$ & $1.71 \pm 0.05$ & $1.48 \pm 0.03$ \\
\hline $22: 6 n-3(D H A)$ & $2.89 \pm 0.18$ & $13.63 \pm 1.55$ & $21.72 \pm 0.75$ & $32.32 \pm 1.62$ & $35.28 \pm 1.18$ & $38.41 \pm 0.46$ & $45.21 \pm 1.13$ \\
\hline $24: 0$ & $1.74 \pm 0.13$ & $1.12 \pm 0.06$ & $0.98 \pm 0.01$ & $0.87 \pm 0.02$ & $0.68 \pm 0.03$ & $0.63 \pm 0.02$ & $0.53 \pm 0.01$ \\
\hline $24: 1 n-9$ & $0.64 \pm 0.03$ & $0.35 \pm 0.01$ & $0.29 \pm 0.01$ & $0.18 \pm 0.02$ & $0.11 \pm 0.01$ & $0.14 \pm 0.05$ & $0.24 \pm 0.02$ \\
\hline
\end{tabular}

$\mathrm{AA}=$ arachidonic acid; $\mathrm{DHA}=$ docosahexaenoic acid; DPA = docosapentaenoic acid; EPA = eicosapentaenoic acid; FA = fatty acid.

* U87-MG cells were supplemented with varying doses of DHA for 24 hours under standard tissue culture conditions prior to analysis. Fatty acid quantification was based on the use of an internal standard administered to the total lipid extract and normalized to protein content. Results are expressed as the mean percentage of targeted fatty acids \pm SD of 3 determinants. 
TABLE 2. Absolute quantities of targeted fatty acids in DHA-supplemented U87-MG cells*

\begin{tabular}{|c|c|c|c|c|c|c|c|}
\hline FA & Vehicle & $10 \mathrm{mM}$ & $20 \mathrm{mM}$ & $30 \mathrm{mM}$ & $40 \mathrm{mM}$ & $50 \mathrm{mM}$ & $60 \mathrm{mM}$ \\
\hline $12: 0$ & $0.15 \pm 0.00$ & $0.12 \pm 0.01$ & $0.09 \pm 0.01$ & $0.10 \pm 0.01$ & $0.10 \pm 0.01$ & $0.09 \pm 0.01$ & $0.09 \pm 0.00$ \\
\hline $14: 0$ & $2.08 \pm 0.23$ & $2.14 \pm 0.04$ & $2.34 \pm 0.14$ & $2.39 \pm 0.12$ & $2.88 \pm 0.06$ & $2.96 \pm 0.13$ & $3.08 \pm 0.06$ \\
\hline 16:0 (palmitic acid) & $32.69 \pm 4.28$ & $33.13 \pm 0.92$ & $32.30 \pm 1.57$ & $33.84 \pm 0.58$ & $45.01 \pm 2.22$ & $46.42 \pm 4.83$ & $50.19 \pm 0.68$ \\
\hline $16: 1 n-7$ & $3.33 \pm 0.39$ & $2.27 \pm 0.03$ & $2.16 \pm 0.13$ & $2.13 \pm 0.12$ & $2.39 \pm 0.03$ & $2.47 \pm 0.07$ & $2.12 \pm 0.36$ \\
\hline 18:0 (stearic acid) & $23.32 \pm 1.98$ & $23.81 \pm 0.93$ & $24.33 \pm 1.28$ & $23.44 \pm 1.39$ & $25.49 \pm 0.24$ & $26.06 \pm 0.80$ & $25.71 \pm 0.63$ \\
\hline 18:1n-9 (oleic acid) & $33.24 \pm 2.82$ & $27.99 \pm 1.01$ & $26.31 \pm 1.43$ & $25.14 \pm 1.47$ & $27.87 \pm 0.21$ & $28.32 \pm 0.85$ & $28.56 \pm 0.76$ \\
\hline 18:2n-6 (linoleic acid) & $5.95 \pm 0.50$ & $5.59 \pm 0.17$ & $5.36 \pm 0.31$ & $5.19 \pm 0.30$ & $5.86 \pm 0.04$ & $5.88 \pm 0.16$ & $6.09 \pm 0.19$ \\
\hline $18: 3 n-6$ & $0.11 \pm 0.01$ & $0.12 \pm 0.00$ & $0.12 \pm 0.01$ & $0.11 \pm 0.01$ & $0.12 \pm 0.00$ & $0.12 \pm 0.01$ & $0.12 \pm 0.00$ \\
\hline $18: 3 n-3$ & $0.12 \pm 0.01$ & $0.09 \pm 0.02$ & $0.15 \pm 0.01$ & $0.16 \pm 0.01$ & $0.23 \pm 0.04$ & $0.24 \pm 0.05$ & $0.20 \pm 0.01$ \\
\hline $18: 4 n-3$ & $0.83 \pm 0.09$ & $0.56 \pm 0.02$ & $0.52 \pm 0.04$ & $0.58 \pm 0.03$ & $0.62 \pm 0.03$ & $0.68 \pm 0.01$ & $0.67 \pm 0.06$ \\
\hline $20: 0$ & $0.44 \pm 0.04$ & $0.37 \pm 0.02$ & $0.37 \pm 0.02$ & $0.39 \pm 0.02$ & $0.45 \pm 0.02$ & $0.44 \pm 0.04$ & $0.50 \pm 0.05$ \\
\hline $20: 4 n-6(A A)$ & $11.93 \pm 0.90$ & $10.89 \pm 0.47$ & $10.88 \pm 0.49$ & $10.42 \pm 0.63$ & $11.05 \pm 0.08$ & $11.52 \pm 0.36$ & $11.29 \pm 0.28$ \\
\hline $20: 5 n-3$ (EPA) & $0.81 \pm 0.01$ & $1.33 \pm 0.07$ & $2.35 \pm 0.15$ & $2.63 \pm 0.13$ & $3.07 \pm 0.02$ & $3.22 \pm 0.08$ & $3.29 \pm 0.07$ \\
\hline $22: 0$ & $0.48 \pm 0.02$ & $0.39 \pm 0.02$ & $0.36 \pm 0.01$ & $0.36 \pm 0.01$ & $0.39 \pm 0.01$ & $0.38 \pm 0.02$ & $0.37 \pm 0.02$ \\
\hline $22: 4 n-6$ & $3.92 \pm 0.20$ & $3.69 \pm 0.17$ & $3.76 \pm 0.16$ & $3.99 \pm 0.22$ & $3.94 \pm 0.05$ & $4.23 \pm 0.07$ & $4.39 \pm 0.09$ \\
\hline $22: 5 n-6$ & $0.45 \pm 0.01$ & $0.41 \pm 0.03$ & $0.71 \pm 0.16$ & $1.10 \pm 0.30$ & $1.32 \pm 0.44$ & $1.35 \pm 0.41$ & $1.09 \pm 0.31$ \\
\hline $22: 5 n-3$ (DPA) & $2.91 \pm 0.15$ & $2.93 \pm 0.12$ & $3.35 \pm 0.14$ & $3.51 \pm 0.18$ & $3.69 \pm 0.05$ & $3.89 \pm 0.10$ & $3.88 \pm 0.07$ \\
\hline 22:6n-3 (DHA) & $3.74 \pm 0.23$ & $18.59 \pm 2.12$ & $32.56 \pm 1.12$ & $56.02 \pm 2.80$ & $74.22 \pm 2.49$ & $87.34 \pm 1.04$ & $118.55 \pm 2.96$ \\
\hline $24: 0$ & $2.25 \pm 0.17$ & $1.53 \pm 0.08$ & $1.46 \pm 0.02$ & $1.50 \pm 0.03$ & $1.43 \pm 0.06$ & $1.44 \pm 0.04$ & $1.40 \pm 0.02$ \\
\hline $24: 1 n-9$ & $0.83 \pm 0.04$ & $0.47 \pm 0.02$ & $0.43 \pm 0.01$ & $0.32 \pm 0.03$ & $0.23 \pm 0.03$ & $0.32 \pm 0.12$ & $0.62 \pm 0.05$ \\
\hline Total FA content & 129.59 & 136.40 & 149.91 & 173.30 & 210.37 & 227.37 & 262.23 \\
\hline
\end{tabular}

* U87-MG cells were supplemented with varying doses of DHA for 24 hours under standard tissue culture conditions prior to analysis. Fatty acid quantification was based on the use of an internal standard administered to the total lipid extract and normalized to protein content. Results are expressed as the mean quantity (mg/mg protein) of targeted fatty acids \pm SD of 3 determinants.

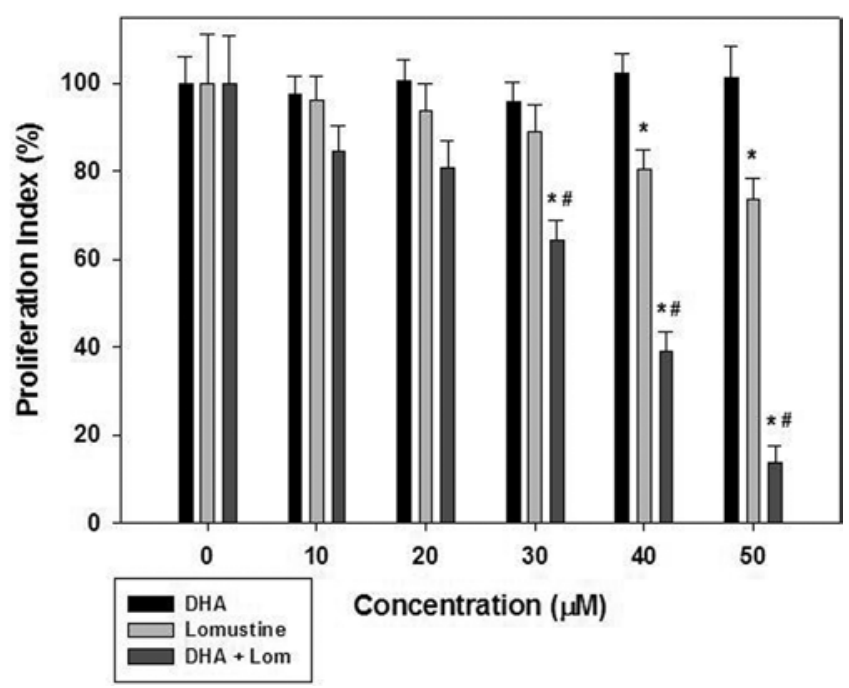

FIG. 2. Synergistic growth inhibitory effect of the combination of DHA and lomustine on U87-MG cells. Growth inhibition was assessed as previously described using a WST-1 proliferation assay. Each concentration represents individual doses of DHA and lomustine alone or in combination. Results are expressed as the mean \pm SD of 4 determinants. Data are analyzed using the Student t-test with Bonferroni correction and the significance differences are reported at $p \leq 0.017$. ${ }^{*}$ Significant difference between treatments and DHA alone. \#Significant difference between combination and lomustine. of lomustine and DHA generated a significant accumulation of cells in the $\mathrm{G}_{2} / \mathrm{M}$ population, which suggests that these cells are arresting in this cell cycle stage. An analysis of cell cycle status following a 24-hour treatment showed an apparent progression through the synthesis phase in lomustine and combination-treated U87-MG cells, while DHA and vehicle-treated cell populations mirrored each other (data not shown).

TABLE 3. Effect of DHA and lomustine supplementation on U87-MG cell apoptosis/necrosis*

\begin{tabular}{lccc}
\hline \multirow{2}{*}{ Agent \& Concentration } & \multicolumn{3}{c}{ Apoptosis/Necrosis Detection (mean \% $\pm \mathrm{SD}$ ) } \\
\cline { 2 - 4 } & Viable & Apoptotic & Necrotic \\
\hline Vehicle (1\% EtOH) & $90.2 \pm 1.5$ & $7.0 \pm 1.1$ & $2.8 \pm 0.5$ \\
\hline $\mathrm{DHA}, 30 \mathrm{mM}$ & $87.1 \pm 1.9$ & $8.0 \pm 1.2$ & $5.0 \pm 0.7$ \\
\hline $\mathrm{DHA}, 40 \mathrm{mM}$ & $86.4 \pm 1.2$ & $7.4 \pm 1.2$ & $6.2 \pm 0.1 \dagger$ \\
\hline Lomustine, $30 \mathrm{mM}$ & $82.4 \pm 1.2 \dagger$ & $10.1 \pm 0.7 \dagger$ & $7.5 \pm 0.5 \dagger$ \\
\hline Lomustine, $40 \mathrm{mM}$ & $76.8 \pm 1.9 \dagger$ & $12.9 \pm 0.7 \dagger$ & $10.3 \pm 1.2 \dagger$ \\
\hline DHA + lomustine, $30 \mathrm{mM}$ & $75.1 \pm 1.9 \dagger$ & $10.6 \pm 0.3 \dagger$ & $14.3 \pm 1.5 \dagger$ \\
\hline DHA + lomustine, $40 \mathrm{mM}$ & $23.1 \pm 6.3 \dagger$ & $18.8 \pm 0.6 \dagger$ & $58.1 \pm 5.7 \dagger$ \\
\hline
\end{tabular}

* U87-MG cells were supplemented with DHA, lomustine, or a combination for 48 hours prior to analysis. Annexin V FLUOS and propidium iodide stains differentiated apoptotic from necrotic cells, whereas nonlabeled cells indicated sustained viability. Results are based on the total cell population and represent the mean $\pm S D$ of 3 determinations. Data were analyzed using the Student t-test with Bonferroni correction.

$\dagger$ Significant difference $(p \leq 0.017)$. 
U87-MG

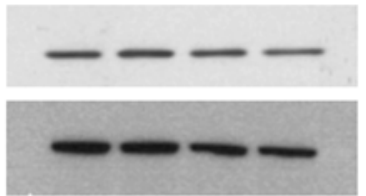

$-1$
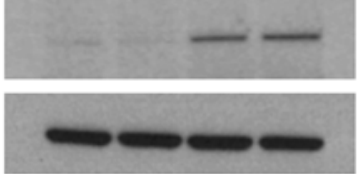

Cleaved-PARP

GAPDH

\section{DHA}

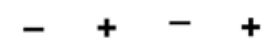

\section{Lomustine}

FIG. 3. Effect of DHA and lomustine supplementation on U87-MG apoptotic intracellular indicators. Treated U87-MG cell lysates were prepared as described in Methods. Electrophoretically separated and transferred proteins were stained with antibodies that recognized naive caspase- 3 and PARP as well as cleaved PARP. GAPDH served as the loading control. Western blots are shown and are representative samples of 3 experiments.

\section{Effect of DHA and Lomustine on Alternative Cell Lines}

To ascertain the clinical relevance and cell specificity of the above results, we next determined the effects of the combined treatment on primary patient glioblastoma cell lines DB029 and MHBT161 as well as a normal primary cell line, human cerebral cortex microvascular endothelial cells (HCCMECs). Figure 4A depicts the effect of lomustine alone or in combination with DHA at varying doses following 24-hour incubation. Although neither lomustine nor DHA dramatically inhibits the DB029 cell line at a $50-\mu \mathrm{M}$ dose, the combination of the two virtually eliminates the proliferative potential of these cells. There is a modest inhibitory effect with increasing concentrations of lomustine; furthermore, the inhibitory effect is enhanced with the addition of DHA, particularly at the $50-\mu \mathrm{M}$ dose. Similar observations were made in the glioblastoma cell line MHBT161 (Fig. 4B). Although an enhanced effect was apparent with nearly every combination of DHA and lomustine, the overall extent of the growth inhibition was not as comprehensive as determined in the U87-MG and

TABLE 4. Effect of DHA and lomustine supplementation on U87-MG cell cycle status*

\begin{tabular}{lccc}
\hline \multirow{2}{*}{ Variable } & \multicolumn{3}{c}{ Cell Cycle Stage (mean \% of cells $\pm \mathrm{SD}$ ) } \\
\cline { 2 - 4 } & $\mathrm{G}_{0} / \mathrm{G}_{1}$ & $\mathrm{~S}$ & $\mathrm{G}_{2} / \mathrm{M}$ \\
\hline Vehicle $(1 \% \mathrm{EtOH})$ & $78.1 \pm 0.9$ & $11.2 \pm 1.0$ & $10.7 \pm 0.2$ \\
\hline $\mathrm{DHA}, 30 \mathrm{mM}$ & $77.7 \pm 0.7$ & $9.4 \pm 1.0 \dagger$ & $13.0 \pm 0.3 \dagger$ \\
\hline Lomustine, $30 \mathrm{mM}$ & $17.8 \pm 1.1 \dagger$ & $6.8 \pm 1.8$ & $75.4 \pm 2.0 \dagger$ \\
\hline $\mathrm{DHA}+$ lomustine, $30 \mathrm{mM}$ & $19.9 \pm 2.1 \dagger$ & $10.7 \pm 2.3$ & $69.4 \pm 3.5 \dagger$ \\
\hline
\end{tabular}

\footnotetext{
* Following synchronization, U87-MG cells were supplemented with DHA, lomustine, or a combination for 48 hours prior to analysis. Propidium iodidelabeled cells were analyzed in a FACSCalibur flow cytometer using MODFit LT software. Results are based on the total viable cell population and represent the mean $\pm S D$ of 3 determinations within each cell cycle stage. Data were analyzed using the Student t-test with Bonferroni correction.

$\dagger$ Significant difference $(p \leq 0.017)$.
}
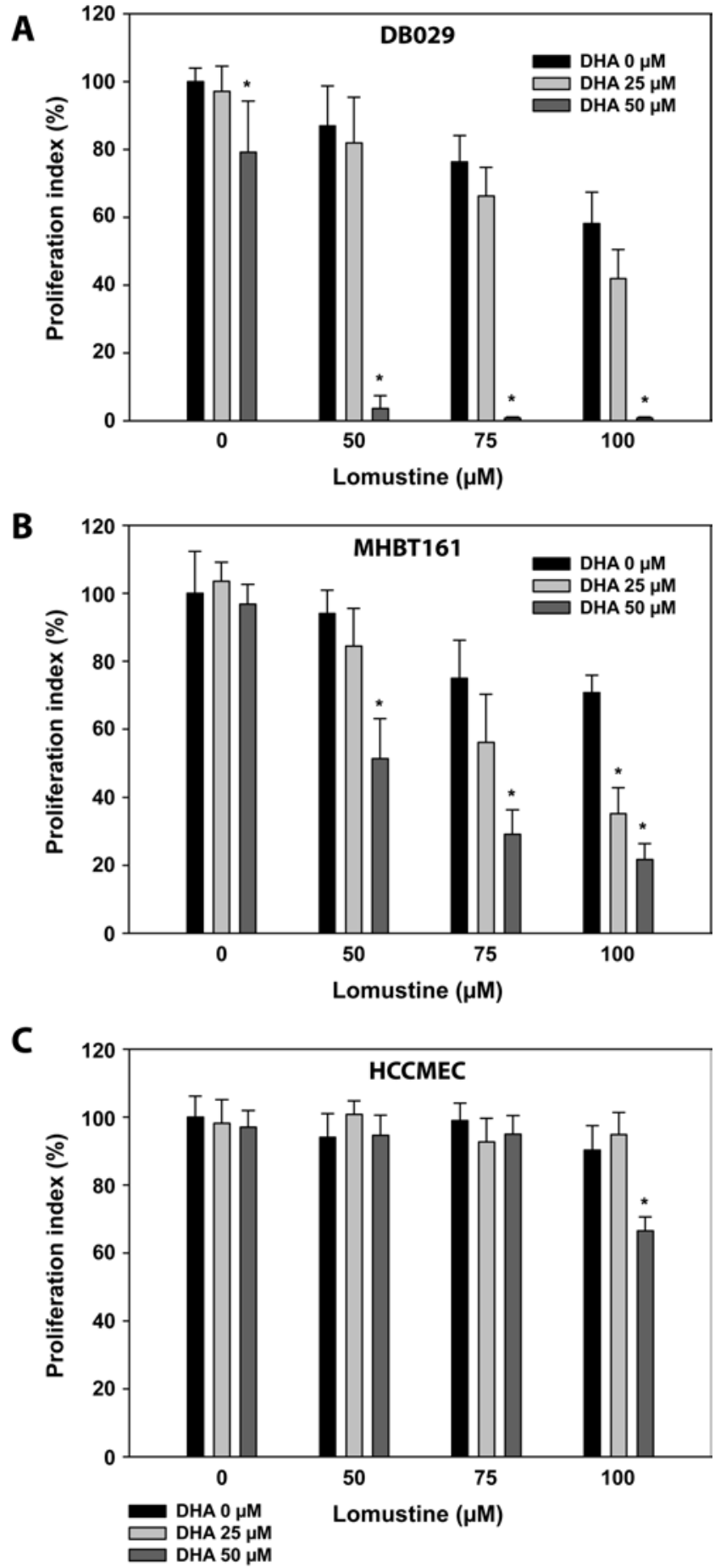

FIG. 4. Effect of DHA and lomustine combinations on growth inhibition. Using the proliferation assay as previously described, the effect of DHA and lomustine, alone or in combination, was assessed on 2 additional human-derived glioblastoma cell lines, DB029 (A) and MHBT161 (B). Human cerebral cortex microvascular endothelial primary cells (C) served as a normal cell phenotype. Results are expressed as the mean \pm standard deviation of 4 determinants. Data were analyzed using the Student t-test with Bonferroni correction. *Values marked with an asterisk reflect significant differences $(p<0.017)$ within a given lomustine data set (i.e., differences seen with increasing concentrations of DHA at a specific concentration of lomustine). 
DB029 cell lines. Figure 4C portrays the effect of these molecules on normal, primary HCCMECs. DHA alone (< $50 \mu \mathrm{M})$ had no significant impact on growth inhibition, whereas lomustine treatment had only a minimal effect at the highest dose examined in this study $(100 \mu \mathrm{M})$. The only significant inhibition was observed in the $100-\mu \mathrm{M}$ combination of lomustine and DHA.

\section{Discussion}

Although a host of chemotherapeutic reagents have been used to treat or at least attempt to slow the progression of glioblastoma, there is currently relatively little benefit to be gained by the use of therapies in this aggressive form of cancer. Previous reports from our laboratory have extensively described the anticancer activities of DHA in various in vitro cell systems as well as in vivo models., ${ }^{4,514,32,33,35,42}$ Furthermore, our laboratory has reported the added benefit of either using DHA in conjunction with known anticancer compounds or directly conjugating DHA with compounds to significantly enhance its anticancer efficacy. Since DHA is the most abundant PUFA in the brain and has been shown to possess anticancer properties, we hypothesized that combining DHA with a currently used chemotherapeutic reagent would result in significantly greater anticancer potential.

U87-MG cells were supplemented with individual fatty acids in escalating doses to determine the extent of growth inhibition. Although most fatty acids tested had little to no effect, all of the omega-3 PUFAs significantly affected the proliferative potential and they did so in a similar manner (DHA, ED $5068.3 \mu \mathrm{M}$; EPA, ED ${ }_{50} 63.5 \mu \mathrm{M}$; DPA, ED 50 $51.4 \mu \mathrm{M})$, based on data in Fig. 1A; however, our investigation focused on the effects of the most abundant omega-3 PUFA in cerebral tissue, namely DHA. Although our laboratory initially assessed the antiproliferative effects of both temozolomide and lomustine, the latter appeared to be more effective in this in vitro system. Furthermore, DHA did not improve the efficacy of temozolomide when they were used together (data not shown). As a result, this investigation focused only on the effect of lomustine in combination with DHA.

Importantly, in the vehicle samples, the DHA level (as a percentage of fatty acids) was significantly lower than the abundant level present in human glioblastomas, which have been reported to contain DHA levels that are approximately half of the level found in brain tissue. ${ }^{16,22,30,40}$ Since the media and serum contain virtually no DHA, it is not surprising that cultured cells tend to reflect the loss of these omega-3 fatty acids. Furthermore, the glioblastoma cell lines used in this investigation underscore the acceptability of these cells to incorporate and tolerate significant quantities of DHA. In stark contrast, cell lines derived from non-neurological sources do not tolerate excess DHA incorporation.

By combining DHA with lomustine, enhanced inhibition of U87-MG cell growth was observed. These effects occurred despite the ability of these cells to tolerate the incorporation of DHA, thus suggesting that DHA, additively or synergistically, enhances the effect of lomustine without compromising cell integrity when administered alone. Lomustine, alone and in combination with DHA, clearly initi- ates an apoptotic cascade as evidenced by flow cytometric analysis. Although the changes in detection of increased cleaved caspase 3 and PARP are subtle, the data are indicative of apoptotic signaling cascades and complement the flow cytometry results. These data strongly support the growth inhibition evidence shown in Fig. 1. Although the U87-MG cells' growth arrested in the $\mathrm{G}_{2} / \mathrm{M}$ phase, the effect appeared to be lomustine-specific, since no significant difference was detected between the results with respect to growth cycle arrest in the presence and absence of DHA. Lomustine administration may ultimately result in the demise of glioblastoma cell lines in vitro; however, when used in combination with DHA, the effect on cell viability is more immediate and dramatic. It is possible that DHA enhances the uptake of lomustine by modulating plasma membranes in glioblastomas. Several studies have shown that DHA incorporation changes the chemical and physical properties of cell membrane phospholipids and enhances uptake of various anticancer drugs, including vincristine in a drug-resistant neuroblastoma cell line, ${ }^{13}$ doxorubicin in mouse leukemia p388 and drug resistant p388/DOX cell lines, ${ }^{21}$ and clioquinol in Raji, SiHa, and MDA-MB-231 cell lines. ${ }^{6}$ In addition, other studies have shown that DHA incorporation causes excessive lipid peroxidation and makes tumor cells prone to destruction. For example, the synergistic effects of DHA and arsenic acid in Daudi, SH-1, SK-Br-3, HT-29, SW-620, and PC-3 cell lines $^{3}$ and the synergistic effects of DHA and paclitaxel in BT-474 and SK-BR-3 cell lines ${ }^{25}$ have been linked to lipid peroxidation. Furthermore, DHA has also been shown to directly modulate the activities of intracellular mediators involved in cell survival and apoptosis including NFKB, PPAR $\alpha$, MAP kinase, AKT, COX-2, Bcl2, and Bax. ${ }^{47}$ The signaling mechanism or mechanisms specifically associated with the combined effect of lomustine and DHA have yet to be elucidated and will be the focus of future studies. Both DHA ${ }^{11,29}$ and lomustine ${ }^{1}$ have been well characterized for their abilities of crossing the blood-brain barrier; therefore, several strategies can be used for delivering them to brain tumors. For example, lomustine can be encapsulated in DHA-containing microsomes, or DHA and lomustine can be chemically linked for delivery into the brain. We will be testing these strategies and investigating the uptake of these agents as a part of our future in vivo studies.

Promising anticancer therapies are always approached with cautious optimism. Often the therapeutic treatment is excessively toxic to normal tissue or effective only on a particular phenotype or mutation. Our laboratory tested the effectiveness of lomustine, alone and in combination with DHA, on cells from a second human-derived glioblastoma cell line, DB029. Although lomustine alone imparted a modest growth inhibitory effect, the combination of lomustine and DHA created an enhanced inhibitory response very similar to that observed in the U87-MG cell line. Importantly, when this experimental design was applied to cells from the normal primary human cerebral cortex endothelial cell line, a significant decrease in the proliferative potential was observed only at the $100-\mu \mathrm{M}$ combination dosage. These data suggest that the effect of the drug and omega-3 fatty acid combination has a certain degree of specificity, especially since this form of treat- 
ment was well tolerated in a normal adjacent cell type. We chose human brain microvascular endothelial cells as a representation of normal cells. DHA and lomustine have to cross through tight junctions of endothelial cells, and therefore these cells are likely to get exposed to DHA and lomustine before cells of any other type. However, it is possible that other neighboring cells such as astrocytes and pericytes are more sensitive to DHA and lomustine. We are currently working on establishing a blood-brain barrier model using co-cultures of endothelial cells, pericytes, and astrocytes for additional experiments.

The present study used concentrations of DHA and lomustine in the 0 - to $100-\mu \mathrm{M}$ range. Based on our study, a combination of 30- $\mu \mathrm{M}$ DHA and $30-\mu \mathrm{M}$ lomustine significantly inhibited proliferation of U87-MG glioblastoma cells. The effective concentrations of these agents are physiologically relevant. The concentration of omega-3 PUFAs after consuming a moderate to high amount of fish for several months can raise plasma omega-3 PUFA levels to $200-400 \mu \mathrm{M}$ without any sign of toxicity. ${ }^{18,29}$ Other in vitro studies found lomustine concentrations greater than $50 \mu \mathrm{M}$ to be effective for glioblastoma cells. ${ }^{31,46}$ Lomustine rapidly degrades under physiological concentration and no intact drug has been detected in serum after oral administration. However, metabolites of lomustine can be detected in the 3 - to $10-\mu \mathrm{M}$ range in plasma and tumor. ${ }^{15}$ Higher concentrations of lomustine are associated with a number of adverse effects, including hematological, renal, hepatic and pulmonary toxicities, and induction of secondary malignancies. Our data suggest that combining lomustine with DHA improved the drug's efficacy, and therefore the combination could potentially limit its toxic effects.

\section{Limitations of Our Study}

This study had several limitations. The experiments were performed under an in vitro condition, which does not entirely represent the microenvironment of the brain tissue. Therefore, doses and efficacy of DHA and lomustine for glioblastoma cell lines may be different from those for tumors growing in the brain. We tested the concept of improving lomustine efficacy by combining it with DHA in a few representative glioblastoma cell lines. Our findings may not be applicable to all glioblastomas. Furthermore, DHA may or may not improve efficacy of other chemotherapeutic drugs that are currently being used. To address these limitations, in vivo studies in orthotopic xenograft models of glioblastoma are required, using combinations of different chemotherapeutic drugs along with DHA. These in vivo studies are the next step in our research.

\section{Conclusions}

Our investigation demonstrated a highly effective combination therapy in an in vitro glioblastoma cell model. The DHA and lomustine combination potently inhibited cancer cell growth and induced apoptosis and necrosis. Future studies should address the mechanism by which this combination imparts this possible synergistic effect. It is quite plausible that DHA sensitizes the glioblastoma cells to the lomustine-induced apoptotic effects through excessive lipid oxidation, enhanced lomustine uptake, or directly modulating activity of mediators of cell survival and apoptosis pathways. Additionally, an in vivo experimental glioblastoma model should be pursued to determine if this efficacy can be translated into a whole organism.

\section{Acknowledgments}

We thank Colin Terry, MS, for statistical analysis and Elaine Bammerlin, MA, for editorial assistance.

\section{References}

1. Bartzatt R: Lomustine analogous drug structures for intervention of brain and spinal cord tumors: the benefit of in silico substructure search and analysis. Chemother Res Pract 2013:360624, 2013

2. Batchelor TT, Mulholland P, Neyns B, Nabors LB, Campone $\mathrm{M}$, Wick A, et al: Phase III randomized trial comparing the efficacy of cediranib as monotherapy, and in combination with lomustine, versus lomustine alone in patients with recurrent glioblastoma. J Clin Oncol 31:3212-3218, 2013

3. Baumgartner M, Sturlan S, Roth E, Wessner B, BachleitnerHofmann T: Enhancement of arsenic trioxide-mediated apoptosis using docosahexaenoic acid in arsenic trioxide-resistant solid tumor cells. Int J Cancer 112:707-712, 2004

4. Berg JP, Glattre E, Haldorsen T, Høstmark AT, Bay IG, Johansen AF, et al: Longchain serum fatty acids and risk of thyroid cancer: a population-based case-control study in Norway. Cancer Causes Control 5:433-439, 1994

5. Connolly JM, Gilhooly EM, Rose DP: Effects of reduced dietary linoleic acid intake, alone or combined with an algal source of docosahexaenoic acid, on MDA-MB-231 breast cancer cell growth and apoptosis in nude mice. Nutr Cancer 35:44-49, 1999

6. Ding WQ, Liu B, Vaught JL, Palmiter RD, Lind SE: Clioquinol and docosahexaenoic acid act synergistically to kill tumor cells. Mol Cancer Ther 5:1864-1872, 2006

7. Elsherbiny ME, Emara M, Godbout R: Interaction of brain fatty acid-binding protein with the polyunsaturated fatty acid environment as a potential determinant of poor prognosis in malignant glioma. Prog Lipid Res 52:562-570, 2013

8. Faragó N, Fehér LZ, Kitajka K, Das UN, Puskás LG: MicroRNA profile of polyunsaturated fatty acid treated glioma cells reveal apoptosis-specific expression changes. Lipids Health Dis 10:173, 2011

9. Folch J, Lees M, Sloane Stanley GH: A simple method for the isolation and purification of total lipides from animal tissues. $\mathbf{J}$ Biol Chem 226:497-509, 1957

10. Freund Levi Y, Vedin I, Cederholm T, Basun H, Faxén Irving G, Eriksdotter M, et al: Transfer of omega-3 fatty acids across the blood-brain barrier after dietary supplementation with a docosahexaenoic acid-rich omega-3 fatty acid preparation in patients with Alzheimer's disease: the OmegAD study. J Intern Med 275:428-436, 2014

11. Glas M, Happold C, Rieger J, Wiewrodt D, Bähr O, Steinbach $\mathrm{JP}$, et al: Long-term survival of patients with glioblastoma treated with radiotherapy and lomustine plus temozolomide. J Clin Oncol 27:1257-1261, 2009

12. Harvey KA, Xu Z, Whitley P, Davisson VJ, Siddiqui RA: Characterization of anticancer properties of 2,6-diisopropylphenol-docosahexaenoate and analogues in breast cancer cells. Bioorg Med Chem 18:1866-1874, 2010

13. Ikushima S, Fujiwara F, Todo S, Imashuku S: Effects of polyunsaturated fatty acids on vincristine-resistance in human neuroblastoma cells. Anticancer Res 11:1215-1220, 1991

14. Kaizer L, Boyd NF, Kriukov V, Tritchler D: Fish consumption and breast cancer risk: an ecological study. Nutr Cancer 12:61-68, 1989

15. Kastrissios H, Chao NJ, Blaschke TF: Pharmacokinetics of high-dose oral CCNU in bone marrow transplant patients. Cancer Chemother Pharmacol 38:425-430, 1996 
16. Kökoğlu E, Tüter Y, Yazici Z, Sandikci KS, Sönmez H, Ulakoğlu EZ, et al: Profiles of the fatty acids in the plasma membrane of human brain tumors. Cancer Biochem Biophys 16:301-312, 1998

17. Krakstad C, Chekenya M: Survival signalling and apoptosis resistance in glioblastomas: opportunities for targeted therapeutics. Mol Cancer 9:135, 2010

18. Kuriki K, Nagaya T, Imaeda N, Tokudome Y, Fujiwara N, Sato $\mathrm{J}$, et al: Discrepancies in dietary intakes and plasma concentrations of fatty acids according to age among Japanese female dietitians. Eur J Clin Nutr 56:524-531, 2002

19. Larsson SC, Kumlin M, Ingelman-Sundberg M, Wolk A: Dietary long-chain n-3 fatty acids for the prevention of cancer: a review of potential mechanisms. Am J Clin Nutr 79:935-945, 2004

20. Lepage G, Roy CC: Direct transesterification of all classes of lipids in a one-step reaction. J Lipid Res 27:114-120, 1986

21. Liu QY, Tan BK: Effects of cis-unsaturated fatty acids on doxorubicin sensitivity in P388/DOX resistant and P388 parental cell lines. Life Sci 67:1207-1218, 2000

22. Martin DD, Robbins ME, Spector AA, Wen BC, Hussey DH: The fatty acid composition of human gliomas differs from that found in nonmalignant brain tissue. Lipids 31:1283-1288, 1996

23. Mehrotra A, Nagarwal RC, Pandit JK: Lomustine loaded chitosan nanoparticles: characterization and in-vitro cytotoxicity on human lung cancer cell line L132. Chem Pharm Bull (Tokyo) 59:315-320, 2011

24. Mehrotra A, Pandit JK: Critical process parameters evaluation of modified nanoprecipitation method on lomustine nanoparticles and cytostatic activity study on L132 human cancer cell line. J Nanomed Nanotechnol 3:8, 2012

25. Menendez JA, Lupu R, Colomer R: Exogenous supplementation with omega-3 polyunsaturated fatty acid docosahexaenoic acid (DHA; 22:6n-3) synergistically enhances taxane cytotoxicity and downregulates Her-2/neu (c-erbB-2) oncogene expression in human breast cancer cells. Eur J Cancer Prev 14:263-270, 2005

26. Mita R, Beaulieu MJ, Field C, Godbout R: Brain fatty acidbinding protein and omega-3/omega- 6 fatty acids: mechanistic insight into malignant glioma cell migration. J Biol Chem 285:37005-37015, 2010

27. Ohgaki H, Kleihues P: The definition of primary and secondary glioblastoma. Clin Cancer Res 19:764-772, 2013

28. Ouellet M, Emond V, Chen CT, Julien C, Bourasset F, Oddo S, et al: Diffusion of docosahexaenoic and eicosapentaenoic acids through the blood-brain barrier: an in situ cerebral perfusion study. Neurochem Int 55:476-482, 2009

29. Philibert A, Vanier C, Abdelouahab N, Chan HM, Mergler D: Fish intake and serum fatty acid profiles from freshwater fish. Am J Clin Nutr 84:1299-1307, 2006

30. Reynolds LM, Dalton CF, Reynolds GP: Phospholipid fatty acids and neurotoxicity in human neuroblastoma SH-SY5Y cells. Neurosci Lett 309:193-196, 2001

31. Röhn TA, Wagenknecht B, Roth W, Naumann U, Gulbins E, Krammer PH, et al: CCNU-dependent potentiation of TRAIL/ Apo2L-induced apoptosis in human glioma cells is p53independent but may involve enhanced cytochrome c release. Oncogene 20:4128-4137, 2001

32. Rose DP, Connolly JM: Antiangiogenicity of docosahexaenoic acid and its role in the suppression of breast cancer cell growth in nude mice. Int J Oncol 15:1011-1015, 1999

33. Schloss I, Kidd MS, Tichelaar HY, Young GO, O'Keefe SJ: Dietary factors associated with a low risk of colon cancer in coloured West Coast fishermen. S Afr Med J 87:152-158, 1997

34. Siddiqui RA, Harvey KA, Walker C, Altenburg J, Xu Z, Terry $\mathrm{C}$, et al: Characterization of synergistic anti-cancer effects of docosahexaenoic acid and curcumin on DMBA-induced mammary tumorigenesis in mice. BMC Cancer 13:418, 2013
35. Siddiqui RA, Harvey KA, Xu Z, Bammerlin EM, Walker C, Altenburg JD: Docosahexaenoic acid: a natural powerful adjuvant that improves efficacy for anticancer treatment with no adverse effects. Biofactors 37:399-412, 2011

36. Siddiqui RA, Jenski LJ, Harvey KA, Wiesehan JD, Stillwell W, Zaloga GP: Cell-cycle arrest in Jurkat leukaemic cells: a possible role for docosahexaenoic acid. Biochem J 371:621-629, 2003

37. Siddiqui RA, Jenski LJ, Neff K, Harvey K, Kovacs RJ, Stillwell W: Docosahexaenoic acid induces apoptosis in Jurkat cells by a protein phosphatase-mediated process. Biochim Biophys Acta 1499:265-275, 2001

38. Siddiqui RA, Shaikh SR, Sech LA, Yount HR, Stillwell W, Zaloga GP: Omega 3-fatty acids: health benefits and cellular mechanisms of action. Mini Rev Med Chem 4:859-871, 2004

39. Siddiqui RA, Zerouga M, Wu M, Castillo A, Harvey K, Zaloga GP, et al: Anticancer properties of propofol-docosahexaenoate and propofol-eicosapentaenoate on breast cancer cells. Breast Cancer Res 7:R645-R654, 2005

40. Stein AA, Opalka E, Rosenblum I: Fatty acid analysis of two experimental transmissible glial tumors by gas-liquid chromatography. Cancer Res 25:201-205, 1965

41. Stupp R, Mason WP, van den Bent MJ, Weller M, Fisher B, Taphoorn MJ, et al: Radiotherapy plus concomitant and adjuvant temozolomide for glioblastoma. N Engl J Med 352:987-996, 2005

42. Vogel VG, McPherson RS: Dietary epidemiology of colon cancer. Hematol Oncol Clin North Am 3:35-63, 1989

43. Vredenburgh JJ, Desjardins A, Reardon DA, Peters KB, Herndon JE II, Marcello J, et al: The addition of bevacizumab to standard radiation therapy and temozolomide followed by bevacizumab, temozolomide, and irinotecan for newly diagnosed glioblastoma. Clin Cancer Res 17:4119-4124, 2011

44. Wang F, Bhat K, Doucette M, Zhou S, Gu Y, Law B, et al: Docosahexaenoic acid (DHA) sensitizes brain tumor cells to etoposide-induced apoptosis. Curr Mol Med 11:503-511, 2011

45. Wick W, Puduvalli VK, Chamberlain MC, van den Bent MJ, Carpentier AF, Cher LM, et al: Phase III study of enzastaurin compared with lomustine in the treatment of recurrent intracranial glioblastoma. J Clin Oncol 28:1168-1174, 2010

46. Wick W, Wick A, Schulz JB, Dichgans J, Rodemann HP, Weller M: Prevention of irradiation-induced glioma cell invasion by temozolomide involves caspase 3 activity and cleavage of focal adhesion kinase. Cancer Res 62:1915-1919, 2002

47. Zhuo Z, Zhang L, Mu Q, Lou Y, Gong Z, Shi Y, et al: The effect of combination treatment with docosahexaenoic acid and 5-fluorouracil on the mRNA expression of apoptosis-related genes, including the novel gene BCL2L12, in gastric cancer cells. In Vitro Cell Dev Biol Anim 45:69-74, 2009

\section{Author Contributions}

Conception and design: Harvey, Siddiqui. Acquisition of data: Harvey, $\mathrm{Xu}$, Wang. Analysis and interpretation of data: Cohen-Gadol, Harvey, Siddiqui. Drafting the article: CohenGadol, Harvey. Critically revising the article: Cohen-Gadol, Saaddatzadeh, Pollok, Siddiqui. Reviewed submitted version of manuscript: all authors. Approved the final version of the manuscript on behalf of all authors: Cohen-Gadol.

\section{Correspondence}

Aaron A. Cohen-Gadol, Goodman Campbell Brain and Spine, Indiana University Department of Neurological Surgery, 355 W. 16th St., Ste. 5100, Indianapolis, IN 46202. email: acohenmd@ gmail.com. 